\title{
Transtornos do humor em enfermaria de clínica médica e validação de escala de medida (HAD) de ansiedade e depressão
}

\author{
Mood disorders among medical in-patients: a validation study of the hospital \\ anxiety and depression scale (HAD)
}

\author{
Neury J. Botega, Márcia R. Bio, Maria Adriana Zomignani, Celso Garcia Jr, \\ Walter A. B. Pereira
}

\author{
Departamento de Psicologia Médica e Psiquiatria da Faculdade de Ciéncias Médicas da Universidade \\ Estadual de Campinas (UNICAMP) - Campinas, SP - Brasil
}

\begin{abstract}
Para estimar a prevalência de transtornos do humor, foram utilizadas a entrevista estruturada "Clinica| Interview Schedule" (CIS-R), $\theta$ a escala "Hospital Anxiety and Depression"(HAD) em 78 pacientes internados em uma enfermaria geral de aduttos (43 homens e 35 mulheres, média de idade $=43,2$ anos). Foi encontrada prevalência instantânea de $39 \%$ de transtornos do humor. Dezesseis $(20,5 \%)$ pacientes preencheram critérios para ansiedade, a maioria dos casos sendo de gravidade leve. Vinte e seis (33\%) casos de depressão foram detectados, 7 dos quais de gravidade moderada. Observou-se uma combinaçăo de sintomas de preocupaçāo, depressāo, ansiedade e insónia. A HAD mostrou-se de fácil compreensão pelos pacientes. As subescalas de ansiedade e de depressāo tiveram consisténcia intema de 0,68 e 0,77 , respectivamente. A correlaçāo dos itens com as respectivas subescalas sugere que essas possuem validades convergentes, nāo discriminantes. Com ponto de corte 8/9, a sensibilidade $e$ a especificidade foram $93,7 \%$ e $72,6 \%$, para ansiedade, e $84,6 \%$ e $90,3 \%$, para depressāo. Na prática clínica, a utilizaçāo da HAD poderla auxiliar na detecção de casos de transtornos do humor que necessitam de tratamento.
\end{abstract}

Depressão, epidemiologia. Ansiedade, epidemiologia. Entrevista psiquiátrica padronizada. Pacientes intermados.

\section{Introduçāo}

A frequêencia global de transtomos do humor em pacientes internados no hospital geral varia de $20 \%$ a $60 \%^{11,13}$. A variaçāo nessas cifras depende da população estudada (características sociodemográficas, tipo de enfermidade, gravidade, cronicidade) e de definições metodológicas (critérios de inclusâo, instrumentos de pesquisa, ponto de corte, definição de "caso" e outros).

Dentre os transtornos do humor, as reaçōes de ajustamento constituem o grupo mais prevalente. A exemplo do observado na assistência primária, o padrão mais comum de sintomas é de natureza indiferenciada, compreendendo uma combinação de preocupaçōes excessivas, ansiedade, depressão e insônia ${ }^{1.13}$.
Apesar de causarem considerável sofrimento $e$ implicações clínica ${ }^{15,19.21}$, pelo menos um terço dos pacientes acometidos por transtornos do humor nāo são reconhecidos como tais pelos seus médi$\cos ^{7}$. Além disso, certos sintomas 'vegetativos' (fadiga, insônia, taquicardia, falta de ar, anorexia, diminuição da libido, e outros) podem ser decorrentes tanto de patologia orgânica quanto mental, confundindo o diagnóstico.

Os citados sintomas encontram-se presentes na maioria das escalas de ansiedade e depressão. Em pesquisas epidemiológicas, tal fato pode superestimar a frequêencia dos transtornos afetivos às custas de pacientes que, sem se encontrarem mentalmente enfermos, apresentem sintomas ocasionados pela patologia física. Outra dificuldade é que em hospital geral torna-se difícil diferenciar 
"casos psiquiátricos", notadamente quando se combinam, além do sofrimento psíquico, doenças físicas e problemas sociais.

Para contornar alguns dos problemas metodológicos apontados, alguns autores têm sugerido que transtornos mentais menos graves sejam medidos ao longo de um continuum: "quanto" de transtomo afetjvo um paciente estaria apresentando? Essa alternativa tem sido amplamente utilizada em estudos comunitários e em assistência primária ${ }^{20.26}$. Desenvolvida para esse fim, a "Clinical Interview Schedule" (CIS) é uma entrevista psiquiátrica estruturada ${ }^{3.8}$. Tanto a versão original $^{3}$, quanto a revisada ${ }^{\mathrm{b}}$, têm se mostrado útil entre pacientes de hospital geral ${ }^{1,13}$. Outro instrumento utilizado, no presente estudo, a "Hospital Anxiety and Depression Scale"(HAD), avalia ansiedade e depressão sem recorrer a itens contendo sintomas vegetativos ${ }^{27}$. Por essa razão, tem sido amplamente utilizada para avaliar transtomos do humor em pacientes com doenças físicas.

A quase totalidade das publicações em epidemiologia psiquiátrica em hospital geral vem de países desenvolvidos ${ }^{11.13}$. No Brasil, a presença de serviços de psiquiatria em hospital geral é modesta, e as pesquisas são escassas ${ }^{2}$. Há, portanto, necessidade de estudos capazes de testar instrumentos de pesquisa, bem como de orientar a alocação dos recursos necessários para aprimorar a assistência aos pacientes internados.

O presente estudo tem como objetivos: estimar a prevalência de ansiedade e depressão em uma enfermaria geral de adultos; e determinar a adequação e a validação da escala "Hospital Anxiety Depression" nesse contexto.

\section{Material e Método}

\section{Delineamento da Pesquisa}

Realizou-se estudo transversal de prevalência de ansiedade e de depressão em pacientes admitidos em uma enfermaria de clínica médica. A avaliaçāo psiquiátrica foi conduzida entre 48-72h após a internação, em duas fases: aplicação do instrumento "Hospital Anxiety and Depression Scale"(HAD), no período da manhã; realizaçã̃o de entrevista psiquiátrica estruturada, a "Clinical Interview Schedule - Revised" (CIS-R), no período da tarde do mesmo dia.

$O$ estudo foi realizado em um hospital geral de 600 leitos, localizado numa regiāo com 2,5 milhões de habitantes. Na enfermaria geral de adul- tos, com 30 leitos, são internados pacientes provenientes do ambulatório e pronto-socorro, ou transferidos de outras enfermarias.

O tamanho amostral foi calculado, chegandose a um mínimo de 80 sujeitos. Tomou-se por base estimativa conservadora de prevalência de transtornos psiquiatricos em pacientes internados $(30 \%)$, com intervalo de confiança de $95 \%$.

Foram elegíveis para o estudo todos os pacientes admitidos consecutivamente na enfermaria ao longo de dois meses (novembro-dezembro/1992), exceto casos transferidos de outras enfermarias do hospital. Dos 83 pacientes internados sob essa condição, 5 foram excluídos: um apresentava estado confusional agudo, outro não falava português, um paciente estava em mau estado geral e dois outros tiveram alta antes que a avaliação psiquiátrica se completasse. Assim, um total de 78 pacientes foram avaliados. Nāo houve recusas de participação.

\section{Instrumentos}

\section{"Clinical Interview Schedule - Revised" (CIS-R)}

Trata-se de uma entrevista psiquiátrica estruturada ${ }^{\mathrm{B}}{ }^{10}$, composta de 14 seçōes: sintomas somáticos, fadiga, concentração, alterações do sono, irritabilidade, preocupaçôes com o funcionamento corporal, depressão, idéias depressivas, preocupaçôes, ansiedade, fobias, pânico, compulsôes e obsessōes. Em cada seção duas questões introdutórias averigüam a ocorrência de sintomas durante a última semana. Em caso afirmativo, a frequiência, a intensidade, a persistência e o grau de incômodo ocasionado pelo sintoma passam a ser avaliados.

A pontuação em cada seção varia de 0 a 4 (exceto "idéias depressivas", 0-5). A definiçāo de "caso" deve corresponder ao limite a partir do qual "um médico geral passaria a se preocupar com o estado emocional de seu paciente"s.8. Quando utilizada com pacientes acometidos por doenças orgânicas, as seçōes sobre sintomas corporais, fadiga e preocupações com o funcionamento corporal podem ser omitidas da pontuaçāo final ${ }^{\natural}$. Foi como procedemos, por precauçāo, ainda que alguns desses sintomas pudessem ocorrer por transtornos mentais.

A tradução da CIS-R acompanhou, em linhas gerais, a versāo disponível em português de sua predecessora ${ }^{1,12}$. $\mathrm{O}$ autor senior do presente trabatho participou de cursos de treinamento da CIS e da CIS-R, na instituição onde esses instrumentos foram desenvolvidos. Os três entrevistadores da presente investigação eram quintanistas de medi- 
cina, O treinamento para utilização da CIS-R totalizou 8 horas, e se consistiu de estudo e discussāo do manual de instruções, além de duas entrevistas com pacientes, realizadas pelo psiquiatra (N.J.B.). Após discussão, dirimiram-se dúvidas em relação à pontuaçāo das seçōes. As entrevistas foram realizadas segundo a disponibilidade dos pesquisadores, buscando-se evitar alocaçāo sistemática de pacientes.

\section{"Hospital Anxiety and Depression Scale" (HAD)}

A escala HAD contém 14 questôes do tipo múltipla escolha. Compõe-se de duas subescalas, para ansiedade e depressão, com sete itens cada. A pontuação global em cada subescala vai de 0 a 21 (Anexo).

Essa escala foi primariamente desenvolvida para ser aplicada a "pacientes de serviços não psiquiátricos de um hospital geral" ${ }^{27}$. Suas principais características são: sintomas vegetativos que podem ocorrer em doenças físicas foram evitados; os conceitos de depressão e ansiedade encontramse separados; o conceito de depressão encontra-se centrado na noção de anedonia; destina-se a detectar graus leves de transtornos afetivos em ambientes não psiquiátricos; é curta, podendo ser rapidamente preenchida; ao paciente solicita-se que responda baseando-se em como se sentiu durante a última semana.

Um estudo-piloto realizado com dez pacientes não demonstrou dificuldaddes de entendimento das questōes. Para contornar problemas de baixa escolaridade, as questōes foram lidas em voz alta. Esclare-se que a tradução da HAD para o idioma português foi realizada sob autorizaçāo de seus autores, por psiquiatra com treinamento no Reino Unido. Duas pessoas leigas, bilíngües, tendo o inglês como língua materna, retraduziram o instrumento para o inglês, chegando-se a um resultado final de consenso.

\section{Escala de Gravidade dos Sintomas}

Ao final da CIS-R, a gravidade dos sintomas de ansiedade e de depressão foram avaliados pelo entrevistador, através de duas escalas semelhantes, uma para ansiedade, outra para depressão, com graduação de 0 a 4 :

(0) Ausência de ansiedade [depressão]

(1) Ansiedade [depressão] "subclínica" leve

(2) Ansiedade [depressão] leve, clinicamente significante
(3) Ansiedade [depressāo] moderada

(4) Ansiedade [depressāo] grave

\section{Análise dos dados}

A determinação de 'casos' de ansiedade e de depressão efetuou-se sempre que os dois critérios seguintes fossem preenchidos: (a) pontuaçāo igual ou superior a dois na seção "ansiedade" da CIS-R (no caso de depressão, pontuação igual ou superior a quatro nas seções "depressão" + "idéias depressivas"); e (b) pontuação igual ou superior a 2 na Escala de Gravidade dos Sintomas.

A análise de dados foi feita atravếs do "Statistical Package for the Social Sciences" (SPSS) ${ }^{23}$. O cálculo da correlação entre os itens (r de Spearman, um teste não-paramétrico aplićável a variáveis não-contínuas) e o alfa de Cronbach (para medir a consistência, ou confiabilidade, intema das duas subescalas) seguiram Carmines e Zeller'. Os coeficientes de validação aqui utilizados encontram-se definidos em Clark e McKenzie ${ }^{4}$.

\section{Aspectos éticos}

Após a explicação dos objetivos do estudo, era assegurado ao paciente o direito de nāo-participação, sem que isso alterasse seu tratamento na instituição. Casos de pacientes apresentando transtornos emocionais foram comunicados ao médico assistente, que poderia levar o caso nos grupos de discussāo médico-psicológica, que vinham se realizando rotineiramente na enfermaria, ou solicitar interconsulta psiquiátrica.

\section{Resultados}

\section{Características Demográficas e Clínicas}

A amostra final compreendeu 78 pacientes, $43((55 \%)$ de sexo masculino. A idade, variando de 14 a 81 anos, alcançou média de 43,2 (desviopadrão $(\mathrm{DP})=17,4)$. As principais características sociodemográficas encontram-se na Tabela 1.

Entre os principais problemas clínicos encontrados nos participantes estão as doenças cardiovasculares/hematológicas $(46 \%)$, neoplasias (13\%) e moléstias infecciosas (10\%).

\section{Perfil Sintomatológico}

Os sintomas psiquiátricos mais prevalentes (pontuação $\geq$ em cada seção da CIS-R) foram 
Tabela 1 - Características sociodemográficas de pecientes ( $N=78$ ) intemados en uma enfermaria de clínica médica.

\begin{tabular}{lll}
\hline Caracteristicas & $N$ & $\%$ \\
\hline $\begin{array}{l}\text { Gènero } \\
\text { masculino }\end{array}$ & & \\
$\begin{array}{l}\text { feminino } \\
\text { Idade (anos) }\end{array}$ & 43 & 55 \\
$14-40$ & 35 & 45 \\
$41-81$ & 36 & 46 \\
Educaçāo & 42 & 54 \\
anaifabeto & 16 & 20 \\
$1-4$ anos & 39 & 50 \\
$5+$ anos & 23 & 30 \\
Estado Civil & 14 & 18 \\
solteiro & 50 & 64 \\
casado/co-habitando & 14 & 18 \\
viúvo/separado/divorciado & & \\
Situaçäo Ocupacionai & 29 & 37 \\
empregado & 29 & 37 \\
desempregado & 20 & 26 \\
dona-de-casa & & \\
\hline
\end{tabular}

insônia: (49\% dos pacientes), preocupaçōes $(36 \%)$, depressão $(28 \%)$, ansiedade $(27 \%)$, idéias depressivas (25\%) e irritabilidade (19\%). Esses sintomas estavam igualmente presentes entre os grupos de pacientes, divididos quanto a características sociodemográficas e a transtornos físicos.

Trinta e um (39\%) pacientes apresentavam transtomos do humor (ansiedade e/ou depressão), clinicamente significativos. Desses, 11 apresentavam depressāo e ansiedade conjuntamente, 15 apenas depressāo e 5 apenas ansiedade.

Um total de 16 pacientes $(20,5 \%), 9$ dos quais de sexo masculino, preencheram os critérios para a definiçāo de "casos" de ansiedade. A Tabela 2 divide esses pacientes de acordo com a gravidade dos sintomas e respectiva pontuação média na subescala de ansiedade da HAD. A maioria (14 pacientes) apresentava grau leve de ansiedade.

Tabela 2 - Número de pacientes por nível de gravidade dos sintomas de ansiedade, com respectivas pontuaçōes médlas na subescala de ansiedade da "Hospital Anxiety and Depression Scale"(HAD-A).

\begin{tabular}{|c|c|c|}
\hline $\begin{array}{l}\text { Gravidade da } \\
\text { ansiedade }\end{array}$ & $\begin{array}{c}\text { Número de } \\
\text { casos }\end{array}$ & $\begin{array}{c}\text { Pontuaçāo média } \\
\text { na HAD-A }\end{array}$ \\
\hline $\begin{array}{l}0 \text { - sem sintomas } \\
\text { 1. "subclínico" } \\
\text { 2- leve } \\
\text { 3. moderada } \\
\text { 4- grave }\end{array}$ & $\begin{array}{l}40 \\
22 \\
14 \\
1 \\
1\end{array}$ & $\begin{array}{l}6,2 \\
7,6 \\
11,6 \\
9,0 \\
13,9\end{array}$ \\
\hline Total & 78 & 7,7 \\
\hline
\end{tabular}

Dentre os 26 (33\%) "casos" de depressāo, 12 eram do sexo masculino. Dezenove apresentavam quadro sintomatológico de gravidade leve; $7 \mathrm{de}$ gravidade moderada (Tabela 3 ).

Dentre os 26 pacientes deprimidos, $6(23 \%)$ já haviam procurado assistência médica no passado devido a "nervosismo ou tristeza" e $10(38 \%)$ já haviam tomado psicofármacos.

\section{Escala para Ansiedade e Depressão (HAD)}

$O$ preenchimento da escala necessitou de, aproximadamente, 4 minutos. De modo geral os itens foram bem compreendidos, exceção feita à palavra "pânico", entre alguns pacientes com baixa escolaridade. As pontuaçðes médias nas subescalas de ansiedade e depressāo foram 7,7 (desvio-padrão $=4,3$ ) e 7,3 (desvio-padrão $=4,8$ ), respectivamente.

Tabela 3 - Número de pacientes por nível de gravidade dos sintomas de depressāo, com respectivas pontuaçōes médias na subescala de depressāo da 'Hospital Anxiety and Depression Scale" (HAD-D)

\begin{tabular}{lccc}
\hline $\begin{array}{l}\text { Gravidade da } \\
\text { depressäo }\end{array}$ & & $\begin{array}{c}\text { Número de } \\
\text { casos }\end{array}$ & $\begin{array}{c}\text { Pontuação média } \\
\text { na HAD-D }\end{array}$ \\
O- sem sintomas & & 39 & 4,3 \\
1 - "subclínico" & 13 & 6,5 \\
2- leve & 19 & 11,9 \\
3- moderada & 7 & 12,4 \\
4- grave & - & - \\
Total & 78 & 7,3 \\
\hline
\end{tabular}

A Tabela 4 mostra a correlação existente entre cada item e as pontuaçōes finais nas subescalas de ansiedade e de depressāo. Os alfas de Cronbach para essas subescalas foram 0,68 e 0,77 , respectivamente. A correlação entre a pontuaçăo na subescala e a gravidade dos sintomas, assinalada pelo entrevistador, foi 0,44 para ansiedade e 0,67 para depressão ( $\mathrm{r}$ de Spearman, $\mathrm{P}<0,001$ ).

A Tabela 5 traz os coeficientes de validação, considerando-se 8/9 como ponto de corte para cada subescala. Se este for baixado para $7 / 8$, a sensibilidade e especificidade serão, respectivamente, $93,7 \mathrm{e}$ 54,8 para ansiedade, 84,6 e 86,5, para depressão.

\section{Discussão}

Foi encontrada uma prevalência instantânea de $39 \%$ de transtornos do humor $(20,5 \%$ de "casos" de ansiedade e 33\% de "casos" de depressāo) entre pacientes de uma enfermaria de clínica médica. A HAD, utilizada para avaliar transtornos do hurnor 
Tabela 4 - Correlaçảo (r de Spearman) entre cada item da "Hospital Anxiety and Depression Scale" a a pontuação total nas subescalas de ansiedade (HAD-A) $e$ de depressăo (HAD-D).

\begin{tabular}{lll}
\hline Item & \begin{tabular}{ll} 
Correlação & Correlaçāo \\
com HAD-A & com HAD-D \\
\hline
\end{tabular} \\
\hline
\end{tabular}

Ansiedade

$\begin{array}{lll}\text { 1. Sentir-se tenso ou contraldo } & 0,56^{* *} & 0,30 * * \\ \text { 3. Medo, algo ruim por acontecer } & 0,63 * & 0,25^{* *} \\ \text { 5. Estar cheio de preocupaçżes } & 0,61^{* *} & 0,43 * * \\ \text { 7. Näo ficar à vontade e relaxado } & 0,58^{* *} & 0,40^{* *} \\ \text { 9. Frio na barriga, aperto no peito } & 0,55^{* *} & 0,33^{*} \\ \text { 11. Sentir-se inquieto } & 0,62^{* *} & 0,22 \\ \text { 13.Sensação de entrar em pânico } & 0,53^{* *} & 0,33 *\end{array}$

Depressão

$\begin{array}{lll}\text { 2. Näo sentir gosto pelas coisas } & 0,44^{* *} & 0,79^{* *} \\ \text { 4. Năo rì e năo se divertir mais } & 0,45^{* *} & 0,75^{* *} \\ \text { 6. Nâo se sentir alegre } & 0,30^{*} & 0,67 * \\ \text { 8. Estar lento para pensar e agir } & 0,29^{*} & 0,43 * * \\ \text { 10. Sem interesse pela aparência } & 0,38^{* *} & 0,62^{* *} \\ \text { 12. Não se animar com o futuro } & 0,24 & 0,61 * \\ \text { 14. Năo ter prazer com TV, rádio } & 0,41^{* *} & 0,66 *\end{array}$

$* P=0,01 \quad$ ** $P=0,001$

em situações de comorbidade física, revelou-se pontecialmente útil como instrumento de "screening". Os resultados sugerem que as subescalas de ansiedade e de depressāo possuem validades convergentes, não discriminativas.

Algumas limitações metodológicas do presente estudo devem ser explicitadas. Embora os sujeitos participantes fossem, provavelmente, representativos do total de pacientes internados na referida enfermaria, os resultados podem nāo ser aplicáveis a outros hospitais gerais. $\mathrm{O}$ tamanho amostral foi calculado para estimativa de prevalência de transtornos mentais concomitantes a doenças físicas.

A CIS-R não propicia diagnósticos psiquiátricos formais. A definição de "casos"de ansiedade e de depressāo baseou-se em critérios operacionais. Essa estratégia, no entanto, não é estranha à pesquisa realizada nessa área $a^{4.13}$.

Os achados confirmam a predominância de transtornos do humor leves, marcados por uma combinaçāo de ansiedade, depressāo, preocupaçōes e insônia. Não foi possível precisar com segurança a proporção de pacientes acometidos por transtornos do humor crônicos, diferenciando-os dos casos agudos. Verifica-se, entretanto, que um quarto dos casos detectados já havia procurado médico por causa de "nervosismo ou tristeza", e que aproximadamente $40 \%$ utilizaram psicofármacos no passado.

As subescalas da HAD apresentaram índices de consistência freqüentemente encontrados em instrumentos psiquiátricos. Todavia, estāo longe do recomendável para instrumentos de "screening" $\left(0,8\right.$, segundo Nunnally $\left.{ }^{18}\right)$, bem como do obtido por outros autores que utilizaram a escala ${ }^{9.13 .16}$. O diminuto número de itens em cada subescala e o pequeno número de sujeitos da amostra poderiam ser responsáveis por esse achado. Quando da avaliação da gravidade do transtorno do humor, a HAD correlacionou-se modestamente com 0 grau de ansiedade, nāo passando de um nível aceitável no caso de depressão. Esses resultados, entretanto, devem ser vistos com cautela, dado o limitado número de pacientes em cada categoria de gravidade.

Os itens da HAD correlacionaram-se positivamente com a pontuação total nas respectivas subescalas de ansiedade e de depressão. Houve também correlação significante, embora de menor magnitude, entre a maioria dos itens de cada escala com a alternativa. Essa observação sugere a possibilidade de as escalas possuírem validades convergentes, deixando de discriminar ansiedade de depressão. Esse achado incita pelo menos duas discussōes: a independência dos constructos de ansiedade e de depressão e a adequação dos instrumentos que medem esses transtornos.

A distinçăo entre ansiedade e depressão é muito útil na prática clínica. Pode orientar melhor, por exemplo, o tratamento farmacológico dos sintomas. Sob o ponto de vista teórico, no entanto, é controvertida. Ainda não se definiu se seriam transtomos distintos, se distintos em categoria ou em dimensão $0^{24}$. Estudos populacionais em geral demonstram a correlação entre as duas dimensóes ${ }^{20}$. Sabe-se, também, que a correlação entre escalas costuma ser consideravelmente inflacionada, quando calculada a partir de população que represente a gama completa de gravidade ${ }^{22}$, como o ocorrido no presente estudo.

Instrumentos de avaliação clínica baseados no julgamento do médico costumam diferenciar razoavelmente ansiedade de depressão, uma distinção difícil de se estabelecer com escalas de autoavaliação. Essas últimas, quando examinadas através de análise fatorial, demonstram pouca discriminação entre sintomas de ansiedade e de depressão. ${ }^{.14}$.

Tebela 5 - Coefiicientes de validaçāo para a "Hospital Anxlety and Depression Scale" (HAD-A = subescala de ansiedade; HAD-D = subescala de depressāo; ponto de corte para ambas subescalas $=8 / 9$ ).

\begin{tabular}{lll}
\hline Coeficientes & HAD-A & HAD-D \\
\hline Sensibilidade & $93,7 \%$ & $84,6 \%$ \\
Especlficidade & $72,6 \%$ & $90,3 \%$ \\
Valor preditivo positivo & $48,4 \%$ & $81,5 \%$ \\
Valor preditivo negativo & $97,7 \%$ & $92,1 \%$ \\
Taxa global de má classificaçåo & $25,6 \%$ & $11,5 \%$ \\
\hline
\end{tabular}


Isso tem levado alguns autores a defender a soma de todos os 14 itens da HAD, produzindo uma medida única de morbidade ${ }^{9.25}$. Outros têm demonstrado o valor prático de se considerarem as duas subescala $^{16.17}$. Estudo utilizando análise fatorial, realizado com 568 pacientes com câncer, por exemplo, acusou dois fatores, após rotação oblíqua, que correspondiam às duas subescalas da $\mathrm{HAD}^{16}$. Como seria de se esperar, ansiedade e depressão não eram totalmente independentes nessa população. Foi encontrada uma correlação de 0,50 entre os dois fatores, após a rotaçāo oblíqua ter sido realizada.

\section{Referênclas Bibliográficas}

1. BOTEGA, N. J. Psychiatric morbidity among medical in-patients: a standardized assessment (GHQ-12 and CIS-R) made by 'lay' interviewers in a Brazilian hospital. Soc.Psycbiatry Psycbiat.Epidemiol., 30: 127-31, 1995.

2. BOTEGA, N. J. \& DALGALLARONDO, P. Saúde mental no bospital geral espaço para o psiquico. Sào Paulo, Hucitec, 1993.

3. CARMINES, E.G. \& ZELIER, R. A. Reliability and validity assessment. Beverly Hill, Sage, 1979.

4. CLARKE, D. M. \& MCKENZIE, D. P. Screening for psychiatric mobidity in the general hospital: methols for comparing the validity of different instruments, IntJ.Metbods Psycbiat. Res, 1: 79-87, 1991.

5. GOLDBERG, D.; GOLDBERG, D.P,; COOPER, B.; EASTWOOD, M.R; KEDWARD, H.B. A standardized psychiatric interview for use in community surveys. Brit.J. Prev. Soc. Med., 24: 18-23, 1970.

6. KLEIN, D.G. Endogenomorphic depression. Arch.Gen.Psychiat, 31: 447-54, 1974 .

7. KNIGHTS, E. \& FOLSTEIN, M. F. Unsuspected emotional and cognitive disturbance in medical patients. Ann.Int.Med., 87: 723, 1977.

8. LEWIS, G, \& PELOSI, A. J. Manual of the revised clinical interview scbedule. London, Institute of Psychiatry, 1990.

9. LEWIS, G. Observer bias in the assessment of anxiety and depression. Soc.Psycbiatry Psycbiat. Epidemiol, 26: 265-72, 1991.

10. LEWIS, G.; PELOSI, A. J.; ARAYA R.; DUNN, G. Measuring psychiatric disorder in the community: a standardized assessment for use by lay interviewers. Psychol.Med., 22: 465-86, 1992.

11. LLOYD G. Textbook of general bospital psychia$t r y$. London, Churchill Livingstone, 1991.

12. MARI J.J.; BLAY, S. L.; IACOPONIi, E. Um estudo de confiabilidade da versão brasileira da Clinical Interview Schedule. Bol. Ofic. Sanit. Panan., 100: 77-88, 1986.

13. MAYOL, R. \& HAWTON, K. Psychiatric disorder in the general hospital. Br/.Psycbiat., 149: $172-90,1986$.

14. MENDELS, J; WEINSTEIN, N.; COCHRANE, C. The relationship between depression and anxiety. Arcb.Gen.Psycbiatry., 27: 649-53, 1972
Outros estudos, atualmente em curso, que utilizam a $\mathrm{HAD}$ poderão contribuir para clarear algumas das questôes metodológicas aqui levantadas. Para o clínico que utiliza a $\mathrm{HAD}$, continua útil o raciocínio que toma ansiedade e depressão como constructos separados. A subescala de depressão da $H A D$, por exemplo, baseia-se fortemente na anedonia, considerada uma característica preditiva de boa resposta a antidepressivos ${ }^{6}$. A utilização de um instrumento simples como o HAD poderia revelar casos de transtornos do humor que podem passar despercebidos pela equipe assistencial.

15. MOFFIC, H. S. \& PAYKEL, E.S. Depression in medical in-patients. Br.J.Psycbiat, 126: 34653, 1975.

16. MOOREY, S.; GREER, S.; WATSON, M.; GORMAN, C.; ROWDEN, L.; TUNMORE, R.; ROBERTSONं, B.; BLISS, J. The factor structure and factor stability of the Hospital Anxiety and Depression Scale in cancer patients. Br. J. Psycbiat., 158: 255-9, 1991

17. MUMFORD, D.B.; TAREEN, I.A. K.; BAJWA, M.A.Z.; BHATTI, M.R.; KARIN, R. The translation and evaluation of an Urdu version of the Hospital Anxiety and Depression Scale. Acta Psycbiat. Scand., 83: 81-5, 1991.

18. NUNNALIY, J.C. Psycbometric theory. New York, McGraw Hill, 1978.

19. QUERIDO, A. An investigation into the clinical, social and mental factors determining the results of hospital treatment. Br.J.Prev.Soc, Med., 13: 33-49, 1959.

20. ROSE, G. \& BARKER, D. J. P. What is a case? Dichotorny or continuum? BrMed.J., 2: 873-4, 1978.

21. SARAVAY, S. M.; STEINBERG, M. D.; WEINSCHEL B. POLLACK, S.; ALOVIS, N. Psychological comorbidity and length of stay in the general hospital. Am.J.Psychiatry, 148: 324-9, 1991.

22. SNAITH, R. P. \& TAYLOR, C. M. Rating scales for depression and anxiety; a current perspective. Br/.Clin.Pbamac., 19: 17S-20S, 1985

23. SPSS Inc, SPSS/PC+ 4.O Base Manual. Chicago, 1990.

24. STAVRAKAKI, C. \& VARGO, B. The relationship of anxiety and depression. A review of the literature. Br.J.Psychiatry, 149: 7-16, 1986.

25. WILKINSON, M. J. B. \& BARCZAK, P. Psychiatric screening in general practice: comparison of the General Health Questionnaire and the Hospital Anxiety Depression Scale. J.R.Col. Gen.Pract, 38: 311-3, 1988.

26. WILLIAMS, P.; TARNOPOLSKY, A,; HAND, D. Case definition and case identification in psychiatric epidemiology: review and assessment. Psychol.Med., 13: 907-21, 1980.

27. ZIGMOND, A. S. \& SNAITH R. P. The hospital anxiety and depression scale. Acta Psycbiat. Scand., 67: 361-70, 1983. 


\begin{abstract}
The revised Clinical Interview Schedule (CIS-R) and the Hospital Anxiety and Depression (HAD) Scale were used to estimate the prevalence of mood disorders among 78 consecutive admissions to a general medical ward in a university generai hospital in Brazil (43 males and 35 females; mean age $=43.2 y r$ ). Interviewers also completed a 5 -point symptom severity scales for anxiety and depression. The definition of cases of anxiety [and depression] was based on wo criteria: a. score $\geq 2$ on the CIS-R section of anxiety [ $\geq 4$ on the CIS-R sections of depression and depressive ideas]; and b. score $\geq 2$ on the clinical severity scale for anxiety [score $\geq 2$ on the clinical severity scale for depression]. A $39 \%$ prevalence rate of affective disorders was found. Sixteen (20.5\%) patients met criteria for anxiety, most of the disorders being of mild severity. Twenty-sic patients (33\%) were depressed, 7 of them in a moderate degree. The HAD was easily understood by the patlents. Anxiety and depression subscales had Internal consistency of 0.68 and 0.77 , respectively. At a cut-off point of $8 / 9$ sensibility and specificity were $93.7 \%$ and $72.6 \%$ for anxiety, and $84.6 \%$ and $90.3 \%$ for depression. HAD items correlated positively with the respective subscales. To a lesser degree, they also correlated with the alternative subscale. Our findings confirm the high prevalence of mood disorders among medical in-patients. In climical practice, the HAD may have a useful role in detecting those petients requiring further psychological care.
\end{abstract}

Depression, epidemiology. Anxiety, epidemiology. Mental status schedule. In-patients. 


\section{ANEXO}

Escala de Ansiedade e Depressão para Hospital Geral (HAD)

\section{ESCALA DE ANSIEDADE E DEPRESSĀO - HAD*}

Este questionário ajudará o seu médico a saber como você está se sentindo. Leia todas as frases. Marque com um " $X$ " a tesposta que melhor corresponder a como vocé tem se sentido na útima semana. Não é preciso ficar pensando muito em cada questäo. Neste questionário as respostas espontâneas têm mais valor do que aquelas em que se pensa muito.

Marque apenas uma resposta para cada pergunta

Eu me sinto tenso ou contraido:
() A maior parte do tempo
() Boa parte do tempo
() De vez em quando
() Nunca

Eu ainda sinto gosto pelas mesmas coisas de antes:
( ) Sim, do mesmo jeito que antes
() Não tanto quanto antes
() Só um pouco
() Já não sinto mais prazer em nada

Eu sinto uma espécie de medo, como se alguma coisa ruim fosse acontecer:
( ) Sim, e de um jeito muito torte
() Sim, mas näo tâo forte
() Um pouco, mas isso não me preocupa
() Năo sinto nada disso

Dou risada $\theta$ me divirto quando vejo coisas engraçadas:
( ) Do mesmo jeito que antes
() Atualmente um pouco menos
() Atualmente bem menos
( ) Năo consigo mais

Estou com a cabeça cheia de preocupaçōes:
( ) A maior parte do tempo
( ) Boa parte do tempo
() De vez em quando
() Raramente

Eu me sinto alegre:
() Nunca
( ) Poucas vezes
() Muitas vezes
( ) A maior parte do tempo

Consigo ficar sentado à vontade e me sentir relaxado:
( ) Sim, quase sempre
() Muitas vezes
() Poucas vezes
() Nunca

Eu estou lento para pensar e fazer as coisas:
() Quase sempre
() Muitas vezes
() De vez em quando
() Nunca

Eu tenho uma sensaçảo ruim de medo, como um frio na barriga ou um aperto no estómago:
( ) Nunca
() De vez em quando
() Muitas vezes
( ) Quase sempre 
Eu perdi o interesse em cujdar da minha aparência:
( ) Completamente
( ) Não estou mais me cuidando como eu deveria
( ) Talvez nāo tanto quanto antes
( ) Me cuido do mesmo jeito que antes

Eu me sinto inquieto, como se eu nāo pudesse ficar parado em luggar nenhum:
() Sim, demais
() Bastante
() Um pouco
() Nāo me sinto assim

Fico esperando animado as coisas boas que estão por vir:
( ) Do mesmo jelto que antes
( ) Um pouco menos do que antes
() Bem menos do que antes
() Quase nunca

De repente, tenho a sensação de entrar em pånico:
() A quase todo momento
() Várias vezes
() De vez em quando

() Nāo sinto isso

Cons|go sentir prazer quando assisto um bom programa de televisão, de rádio, ou quando leio alguma coisa:
( ) Quase sempre
() Várias vezes
() Poucas vezes
( ) Quase nunca 